\title{
Aproximaciones teóricas a la relación entre Estado, Medios y Públicos: España, Rusia y China
}

Carlos Manuel Rodríguez Arechavaleta*

DOI: https://doi.org/:10.33571/revistaluciernaga.v12n24a6

\section{Resumen}

Esta revisión bibliográfica aporta elementos teóricos para el análisis de la complejidad de la relación entre los medios, el Estado y los públicos. Permite pensar las dinámicas comunicativas en la España postfranquista, la Rusia post-soviética y la China de transición economía basada en la mercancía y la apertura internacional.

Palabras clave: Prensa; periodismo; medios, públicos; estado.

Recibido. Junio 19, 2020

Aceptado. Julio 06, 2020

*Profesor-Investigador del Departamento de Comunicación de la Universidad Iberoamericana Ciudad de México. Miembro del Sistema Nacional de Investigadores de México - ConacyT, Nivel 1. Orcid: https://orcid. org/0000-0002-1188-2403; e-mail: carlos.rodriguez@ibero.mx

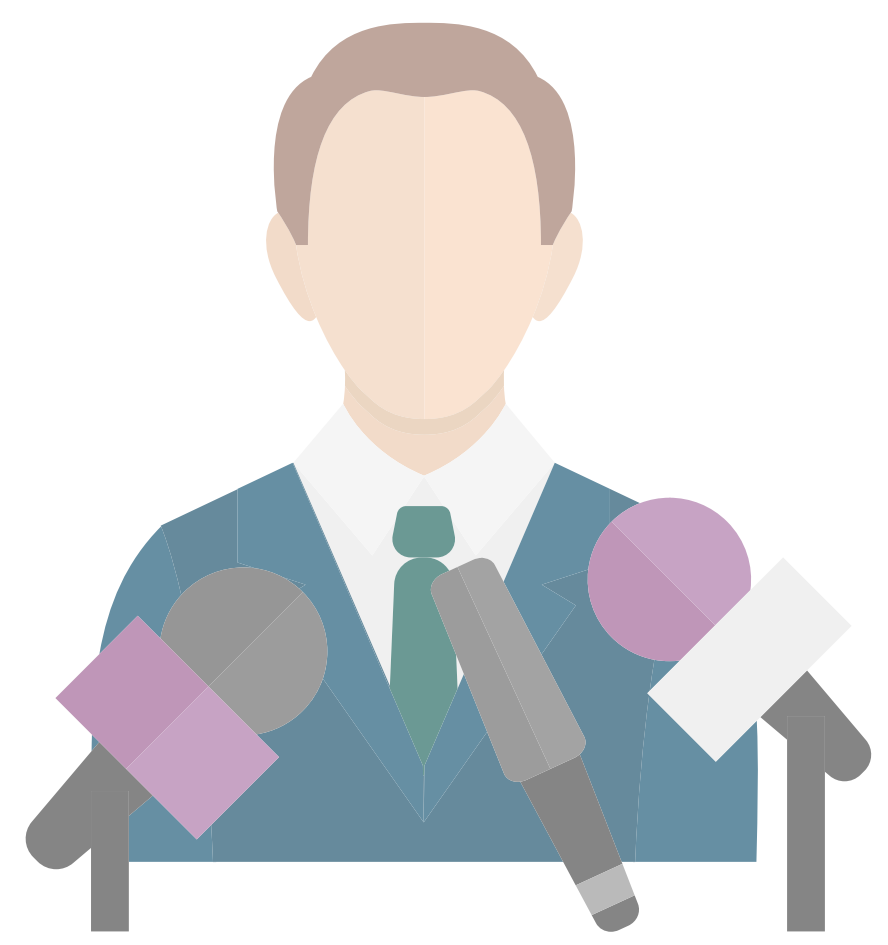




\title{
Theoretical Approaches to the Relationship between State, Media, and Audiences: Spain, Russia and China \\ Carlos Manuel Rodríguez Arechavaleta*
}

DOI: https://doi.org/:10.33571/revistaluciernaga.v12n24a6

\begin{abstract}
This bibliographic review provides theoretical elements for the analysis of the complexity of the relationship between the media, the state, and the audiences. It allows thinking about the communicative dynamics in post-Franco Spain, post-Soviet Russia and the China of transition economy based on merchandise and international openness.
\end{abstract}

Keywords: Press; journalism; media, public; state.

Received. June 19, 2020 Accepted. July 06, 2020

*Professor-Researcher, Department of Communication, Universidad Iberoamericana Ciudad de México. Member of the National System of Researchers of Mexico - ConacyT, Orcid: https://orcid.org/0000-0002-11882403; e-mail: carlos.rodriguez@ibero.mx

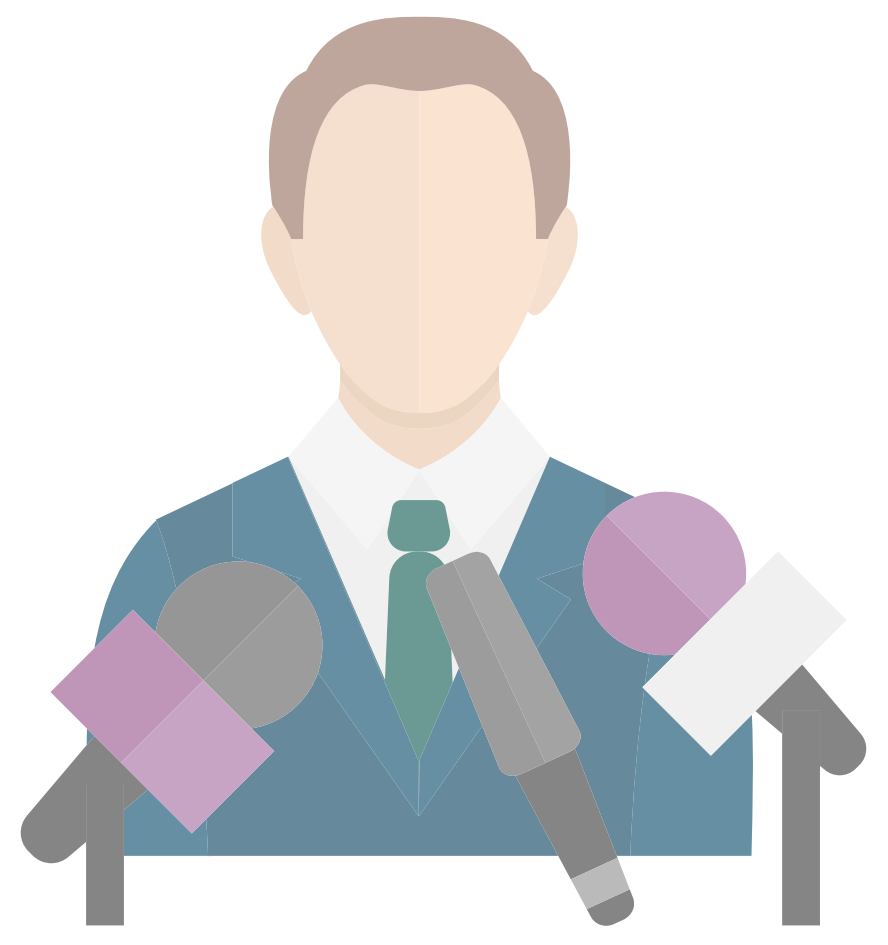




\section{Abordagens teóricas da relação entre Estado, Meios de Comunicação Social e Públicos: Espanha, Rússia e China}

Carlos Manuel Rodríguez Arechavaleta*

DOI: https://doi.org/:10.33571/revistaluciernaga.v12n24a6

\section{Resumo}

Esta revisão bibliográfica fornece elementos teóricos para a análise da complexidade da relação entre os meios de comunicação social, o Estado e o público. Permite-nos pensar na dinâmica comunicativa na Espanha pós-Franco, na Rússia pós-soviética e na China da economia de transição baseada na mercadoria e na abertura internacional.

Palavras chave: Prensa; jornalismo; média, público; estado.

Recebido. Junho 19, 2020

Aceitado. Julho 06, 2020

*Professor-Pesquisador do Departamento de Comunicação da Universidade Iberoamericana Ciudad México. Membro do Sistema Nacional de Investigadores do México - ConacyT, Nível 1. Orcid: https://orcid. org/0000-0002-1188-2403; e-mail: carlos.rodriguez@ibero.mx

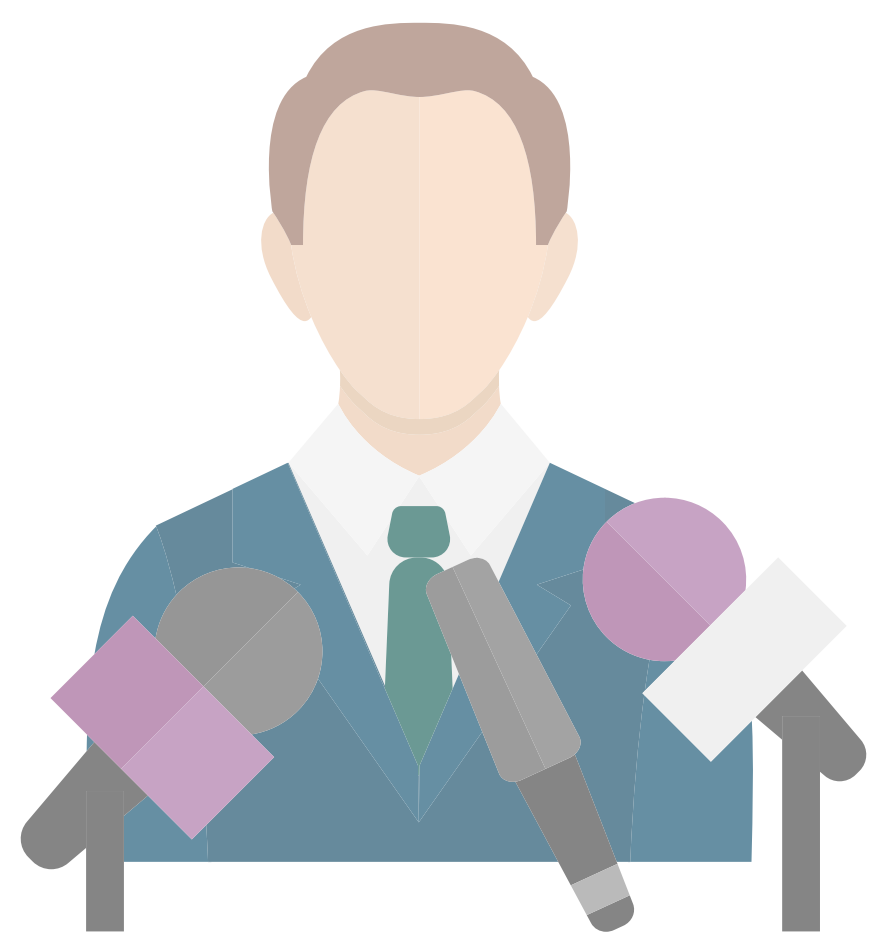




\section{Introducción}

El énfasis normativo de los modelos de prensa en democracias liberales occidentales con economía de mercado ha sido cuestionado por el creciente interés en la relación prensaestado-públicos en países con regímenes autoritarios, híbridos o en procesos de transición política (Zielonka, 2015; Gross \& Jakubowicz, 2013; Hallin \& Mancini, 2012; Voltmer, 2006; Esser \& Pfetsch, 2004; Price \& Rozumilowicz, 2002; Curran \& Jin-Park, 2000).

La conexión entre medios masivos de comunicación, (especialmente, los modelos de periodismo) y la dinámica política (cambio de régimen) a pesar de una prolífica literatura en diversas disciplinas, adolece de un modelo teórico interpretativo preciso. En palabras de J. Downing (1996), las investigaciones sobre el estado, los regímenes totalitarios, los (nuevos) movimientos sociales y las transiciones políticas han prestado poca atención a sus dinámicas comunicativas intrínsecas. Paradójicamente, el mainstream o tendencia de medios y sus diversas expresiones culturales reflejan una dinámica de tensión en el equilibrio de poder entre el estado autoritario y los (nuevos) movimientos sociales que puede derivar en los procesos de transición y consolidación democrática.

Al respecto, Randall (1993) ha llamado la atención sobre el importante rol de los medios en preparar el terreno cultural e ideológico para la democracia, especialmente en países del Tercer Mundo. Al agudizar el descontento popular y la conciencia de alternativas políticas a largo plazo, éstos han acelerado las nuevas dinámicas de comunicación política que han implicado una significativa presión sobre las autoridades.

Investigaciones comparadas han cuestionado el posible efecto causal entre la reforma de medios y los procesos de democratización, así como la conceptualización de los medios masivos y los modelos de periodismo como agentes del cambio social y político o como componentes del status quo de un régimen autoritario/totalitario (Gross \& Jakubowicz, 2013; Voltmer, 2006; Price \& Rozumilowicz, 2002). Por su parte, los estudios de casos, a pesar de reconocer que ciertos ajustes en la estructura de los medios y el comportamiento del escenario político en la historia de un país pueden mover la política hacia el reconocimiento de normas democráticas, apuntan a la interrelación de un complejo set de factores estructurales, culturales y socio-políticos de sociedades concretas. En síntesis, la reforma de medios y la democratización parecen dos procesos excluyentes con escaso o nulo efecto el uno sobre el otro (Price \& Rozumilowicz; 2002).

La evidencia de las transiciones en Rusia y los países de Europa del Este no muestran el ansiado tránsito de un rol periodístico de legitimador ideológico de un estado autoritario/ totalitario a una profesión independiente, cuya función principal es vigilar al estado (watchdog) y generar debate público. Por su otra parte, tanto el periodismo actual como las audiencias reproducen rasgos culturales y prácticas no liberales. La reforma económica China ilustra además la elasticidad adaptativa y funcional de los periodistas y la capacidad de resiliencia de un régimen autoritario. 


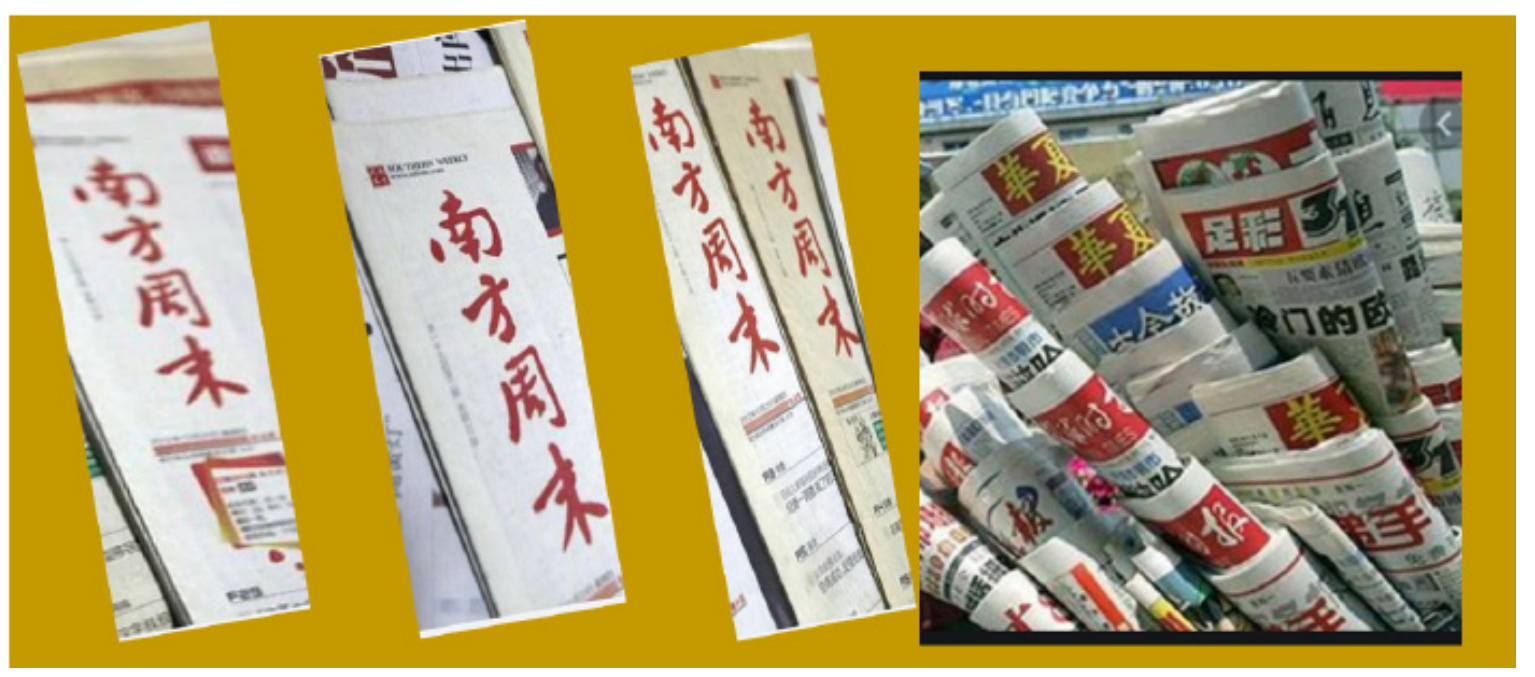

1. La relación medios-estados-públicos en regímenes autoritarios, híbridos o transicionales

Gunther \& Mughan (2000) en su clásica investigación comparada, evadiendo algunos presupuestos simplistas, confirman que los efectos políticos de los medios son variados y altamente condicionados por otros factores institucionales, socio-estructurales y de nivel micro.[1]. Al analizar el impacto de los medios a corto y largo plazo en regímenes autoritarios/ totalitarios, los autores observaron que el estado, a pesar de ejercer un férreo control de los flujos informativos, tuvo una limitada capacidad para configurar las actitudes y orientaciones políticas de sus poblaciones o resocializarlas, garantizando su legitimidad, y finamente, colapsaron.

En efecto, ni Franco ni Pinochet pudieron perpetuar sus regímenes autoritarios, a pesar de sus rígidos controles. La permeabilidad de las fronteras nacionales en Alemania Oriental redujo el costo de acceso de sus ciudadanos a la programación de los canales y la publicidad comercial de Alemania Occidental, la cual contradecía la propaganda estatal sobre la superioridad del socialismo y la crisis del capitalismo. El desarrollo de la industria del turismo fue un recurso importante de exposición a información inconsistente en países de Europa Central, del Este y en España. Por otro lado, factores socio-estructurales como sectores sociales o grupos con algún grado de subcultura autónoma del estado y del resto de la sociedad, pueden potenciar la creación de redes asociativas y conductos de opiniones, actitudes y valores inconsistentes con la propaganda oficial y subversiva respecto a su credibilidad y aceptabilidad.

Desde el punto de vista micro-individual, uno de los factores que afecta la predisposición a aceptar o rechazar los mensajes es la educación, al conducir el desarrollo de normas protoparticipativas y expectativas que enfrentan el rol de sujeto impuesto sobre los individuos en sistemas no democráticos; a mayor experiencia de viajes, lecturas e intercambios con el exterior, mayor será la complejidad cognitiva y mayor la resistencia a los efectos manipulativos de la información del régimen.

Como se planteó en párrafos anteriores, el énfasis normativo de los modelos de prensa en democracias liberales occidentales con economía de mercado ha sido cuestionado por el creciente interés en la relación Prensa-Estado-Públicos en países en desarrollo con 
regímenes autoritarios, híbridos o en proceso de transición política (Zielonka, 2015; Gross \& Jakubowicz, 2013; Hallin \& Mancini, 2012; Voltmer, 2006; Esser \& Pfetsch, 2004; Price \& Rozumilowicz, 2002; Curran \& Jin-Park, 2000).

Estos estudios, a diferencia de los enfoques de la "transitología" que asume el cambio político desde la perspectiva binaria de los autoritarismos a las democracias liberales, proponen comprender la variedad de arreglos de los medios en sus respectivos términos históricos, focalizando los procesos de cambios más que en una visión teleológica de la historia (Meng \& Rantanen, 2015; Roudakova, 2012) [2].

En ese sentido, Roudakova propone estudiar los procesos históricos de (re)articulación de los medios-estados-públicos como resultado de la tensión por el mantenimiento/erosión del orden en un escenario incierto; en otras palabras, su preocupación central son los procesos de reproducción de la legitimidad del Estado a través de los países y los periodos históricos (2012: 262-ss). [3]. Entonces, el rol de los medios será más constitutivo que instrumental, pues no se reducen a plataformas de lucha política, sino que toman activamente parte en producir la dinámica de la política (Meng \& Rantanen; 2015; Roudakova, 2012).

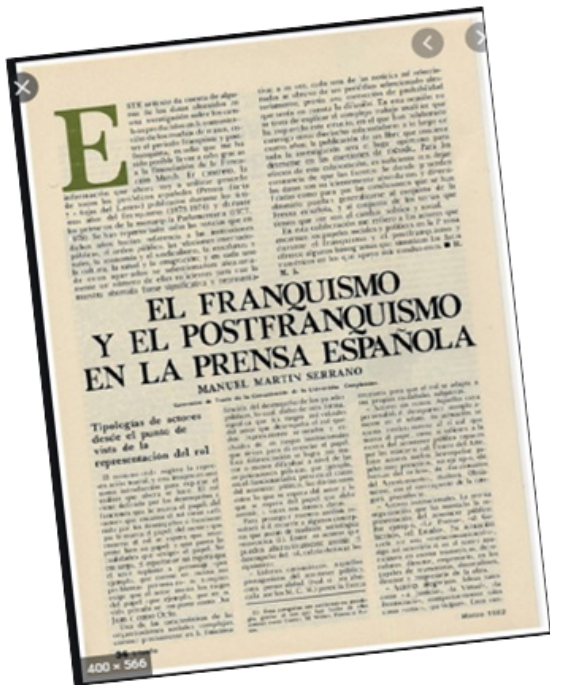

El caso español proyecta efectos contradictorios de la prensa en el periodo de transición democrática. Reig Cruañes (2014) en un interesante trabajo en el que cuestiona "el relato consensual de la transición española" se pregunta si la prensa fue el motor de cambio - o parlamento de papel- que anticipó a la sociedad civil y la opinión pública en la construcción de un espacio público democrático, concluyendo que solo unos pocos medios y unos cuantos periodistas destacaron por su defensa de una evolución inequívocamente democrática.

En sus propias palabras, toda esa formidable industria de la información -heredada del franquismo- resultaba de una uniformidad abrumadora y de un tono casi enteramente consonante [4]. Solo en el primer postfranquismo, o periodo de desconcierto, se manifestó una verdadera prensa conflictual, la cual descubre tardíamente los movimientos sociales y políticos de oposición y se encontró con una sociedad civil ignorada hasta entonces, que se abrió a la oportunidad de distanciarse de los rígidos esquemas del discurso franquista. Fue entonces el diálogo que la prensa estableció con la sociedad civil lo que propició la emergencia de un espacio público democrático (2014: 178). 
Barrera y Zugasti (2006) tienen una visión diferente del rol político de la prensa en la transición española, reconociéndole un importante rol mediador entre las elites políticas y los ciudadanos, al apoyar la mayoría de los periódicos las principales directrices de la reforma política y las estrategias de consenso de los actores políticos. Según estos autores la prensa influyó en el cambio político de dos formas: desde la selección y categorización de las noticias provistas para los lectores (público), y los comentarios editoriales dirigidos más específicamente a las élites políticas; promoviendo, además, tres valores básicos de la cultura política durante la transición: la libertad, la amnistía y la autonomía, principales demandas de las demostraciones de la sociedad civil en esos momentos (2006: 28).[5]. Es destacable su esfuerzo en focalizar las áreas de acuerdos y moderación sobre las posturas divergentes polarizantes de ciertos diarios, así como la progresiva introducción de noticias sobre los nuevos actores políticos en la arena pública.

Otra dinámica no exenta de paradojas y tendencias regresivas fue el transito del modelo de medios Soviéticos (1917-1991) a Neo-Soviético en sus dos principales versiones, "el modelo de cuarto poder" en la liberalización del primer gobierno post-soviético de Boris Yeltsin (1991-1996), al "modelo autoritario-corporativo" implementado a partir de su reelección en 1996. En efecto, al calor de la glasnost impulsada por M. Gorbachov, la figura de B. Yeltsin emerge como un líder emancipador asociado a los valores occidentales del libre mercado y comercio, la libertad de prensa y la negación de la censura, los cuales se plasmaron en la Ley Rusa sobre los Medios Masivos promulgada en diciembre de 1991 y en la Constitución de 1993. Sin embargo, como Presidente de la nueva Federación Rusa, B. Yeltsin gobernó ampliamente por decretos desconociendo los checks and balances horizontales del naciente gobierno ruso, y hacia 1993 comenzó a prohibir los diarios opositores. Peor aún, B. Yeltsin personificó la creencia de que el fin justifica los medios, y medidas no democráticas fueron tomadas para fortalecer la democracia, incluyendo la instrumentalización política del sistema de medios. [6].

V. Putin por su parte, enarboló un discurso nostálgico articulado sobre los valores tradicionales como patriotismo, la grandeza de Rusia, la solidaridad social y el colectivismo, no comunes en el discurso occidental, y pronto reemplazó el discurso de "en nombre de la democracia" de B. Yelstin por el de "en el nombre de las operaciones antiterroristas", justificando el uso de las restricciones a la libertad de prensa y los derechos individuales a la información por la protección del secreto de estado, la seguridad del estado, etc.

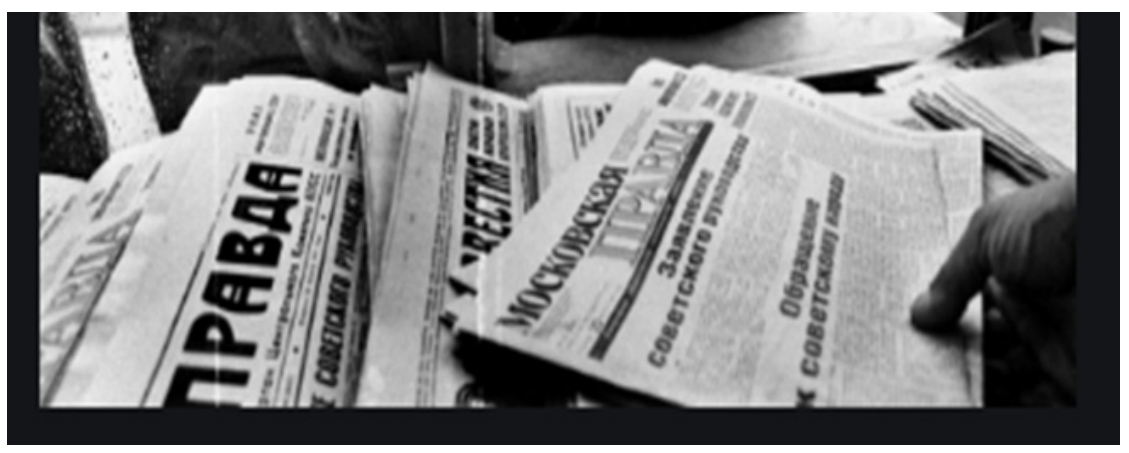


Hedwig de Smaele (2013) encuentra dos paradojas en la situación de medios en Rusia; la primera, vinculada a la esfera legal: a pesar de existir una ley de prensa liberal y una garantía constitucional de la libertad de prensa, Rusia sufre de un poder judicial dependiente y capturado, débil implementación de la ley [7], registro obligatorio de medios masivos, leyes restrictivas sobre difamación y regulaciones antiterroristas, lo que permite una serie de controles de medios por el gobierno a través del Ministerio de Medios, el nombramiento de personal directivo de canales de televisión importantes, además de la dependencia financiera de los subsidios del estado o patrocinadores corporativos semi-estatales, o acceder a facilidades como editoriales, imprentas, acceso a transmisores o satélites, cuya licencia para operar deben obtener de la administración del estado más que de reguladores independientes.

La segunda paradoja se refiere a la experiencia económica rusa y el pluralismo político y la diversidad de propiedad: al carecer de transparencia la propiedad, el estado es un fuerte jugador/propietario en el mercado y los propietarios privados son oligarcas cuyos intereses económicos, políticos y de medios son interdependientes, lo que explica su escasa preocupación por la libertad de prensa, solo dispuestos a intervenir cuando sus intereses están directamente en riesgo (Hedwig de Smaele: 2013).

Pero, además, y más desconcertante aún, es la aceptación por parte de los periodistas y los públicos del uso instrumental de los medios masivos. Los periodistas se conciben a sí mismos como misioneros de ideas antes que como neutrales observadores que presentan realidades. Al aplicar focus group a la audiencia rusa entre 2000 y 2004, a la investigadora Sarah Oates le llamó la atención su rechazo unánime a la idea de "objetividad" y "balance" en los medios masivos, pues éstos eran concebidos como jugadores políticos al servicio de patrocinadores financieros y políticos que actuaban como fuertes filtros políticos de la información.

Al comprender el tipo de filtro, podían comprender la noticia (Oates: 2007). El ciudadano ruso tiene acceso a información pública sobre temas selectos, limitados, no polémicos, de ahí su escasa participación en el debate político y la vida cívica. [8].

La aparente diversidad de medios masivos en Rusia, incluso independientes, no se traduce en autonomía del poder político. Existen instituciones democráticas aparentes, incluyendo elecciones, parlamento, presidente electo popularmente, medios diversos, pero estas instituciones carecen de contenido democrático, y los medios masivos simplemente repiten la fábula de la interacción democrática. El Kremlin ha sofisticado los mecanismos de regulación y control político, apoyado además en la ambivalencia de los marcos regulatorios, lo que se ha traducido en una creciente auto-regulación en las prácticas periodísticas como mecanismo de adaptación y sobrevivencia personal y profesional. Tal vez ello explique la autopercepción del periodista ruso como jugador político, y no como vigilante -watchdogs- o retador del status quo político (Oates; 2007). [9].

Como se puede apreciar, en la Rusia post-soviética posterior a 1992, las reformas de liberalización económica y la competencia política y electoral produjeron un cambio institucional con resultados inciertos, dada la implementación de una democracia 
capitalista cuya principal característica fue la interpenetración entre los emergentes agentes económicos y políticos en la competencia por compartir el capital político. Ambos, empresarios capitalistas y actores políticos, construyeron diferentes alianzas para lograr sus fines (capital económico y ventajas políticas), convirtiéndose en la élite dominante que estableció el control sobre los medios.

Por otro lado, el tránsito de la economía socialista a la economía de mercado no generó un rápido crecimiento económico basado en el consumo que se tradujera en un incremento en el nivel de vida de la sociedad, y el activismo político de los años 80' se transformó en apatía social y valores como el éxito individual y el hedonismo (Vartanova; 2012).

Tampoco la privatización de la industria de medios en 1992, y su reposicionamiento, generaron un modelo más democrático de comunicación política; por el contrario, se incrementó el patrocinio político y el uso manipulativo de la prensa y su creciente sensacionalismo [10]. Como acertadamente reconoce McNair (2000, cit. Vartanova, 2012: 126), "el resultado fue la creación de un sistema de (hiper)capitalismo híbrido", caracterizado por el estado burocrático como principal agente económico y la subsecuente integración del emergente mercado y el estado en un "complejo industrial de medios" que ejerció una determinante influencia sobre las políticas del sistema de medios.

En esas condiciones es entendible el limitado rol de los medios en la construcción de los partidos políticos independientes en Rusia, pues la fuerza crucial en el juego político permaneció en el estado (Vartanova, 2012). Más que paralelismo político (Hallin \& Mancini, 2007), estamos en presencia de relaciones de "clientelismo política-medios" (Roudakova, 2008).

Respecto a la cultura política y mediática, la histórica tradición de subordinación del individuo al estado, entendido éste como fuerza sacra que garantiza la unidad de la nación rusa, y la percepción de los medios como parte esencial de las estructuras de poder la han limitado. La llegada de Vladimir Putin al poder en el 2000 ha acelerado la re-estatización e instrumentalización de los medios para la construcción de una nueva "idea de nación".

No menos importante es la ambigüedad jurídica y su implementación discrecional por el Estado (Hedwig de Smaele, 2013; Oates, 2007), contradicción que ha sido aprovechada por políticos nacionales y locales que amenazan a los medios con el cierre por coberturas adversas (Oates, 2007). Un poder judicial dependiente y capturado permite la aplicación de regulaciones altamente restrictivas y controles para los medios y periodistas no leales al estado (Hedwig de Smaele; 2013) [11], lo que se ha traducido en una creciente autoregulación en las prácticas periodísticas como mecanismo de adaptación y sobrevivencia personal y profesional. Tal vez ello explique la autopercepción del periodista ruso como jugador político y no como watchdogs o retador del status quo político (Oates; 2007).

El declive de un periodismo como profesión creativa ha sido paralelo al nacimiento de una profesión orientada a la publicidad y las relaciones públicas. Vartanova (2012) reconoce la paradójica coexistencia de la independencia de medios, dada la creciente descentralización 
y autonomía formal de las salas editoriales de las agencias estatales y partidos políticos, y el bajo nivel de libertad de discursos por la creciente centralización política, la reestructuración de la esfera pública bajo la nueva "idea nacional" y el consentimiento de la instrumentalización del periodismo, su uso como instrumento de campañas políticas y la autocensura en las oficinas editoriales.

China ofrece otro interesante caso de estudio para entender la lógica entre reforma económica y liberalización del sistema de medios. Las reformas económicas en China inician en 1978 bajo la dirección del nuevo liderazgo de Deng Xiaoping encargado de recuperar la legitimidad del Partido Comunista (PC) posterior a la muerte de Mao Zedong, y al periodo de férreo control ideológico del PC conocido como la Revolución Cultural. La estrategia de debate ideológico seguida por Deng "El Gran Debate relativo a los Criterios de la Verdad" le permitió replantear los principios de la ideología maoísta por el pragmatismo y el empirismo: "buscar la certeza de los hechos" (Chan \& Qui: 2002: 29-ss) y definir como meta central de la política la satisfacción de las aspiraciones materiales del pueblo sin sacrificar el liderazgo del Comité Central del Partido Comunista (CCP).

Para justificar los imperativos económicos y reconciliarlos con el control social, Deng acuñó la frase "socialismo con características Chinas" y se planteó un sistema de reformas en tres partes: la responsabilidad del sistema, la transición a una economía basada en las mercancías, y la apertura a la economía internacional. Sin embargo, hasta finales de los años 80' estas reformas no chocaron con los marcos de la economía socialista, y se condujeron entre periodos de retroceso y de reforma.

El momento determinante para la liberalización económica fue resumido por Deng en 1992 en la frase "construir una economía de mercado socialista con características Chinas". En los siguientes diez años, el mercado se convirtió en un factor constante en la reconfiguración de la estructura de medios como empresarios en relación al partido como supervisor de lo público, pero la orientación de éstos será hacia el consumo (Winfield \& Peng; 2005).

Los investigadores Chan \& Qui (2002) reconocen en la Ley Civil de Provisiones Generales de 1986 incentivos a la autonomía de muchos sectores de la economía, incluyendo los medios. Dicha ley articula lineamientos para la regulación de la economía y las transacciones personales con el presupuesto implícito de que la sociedad está compuesta de discretas entidades, cada una de las cuales puede contraer contratos para vender, comprar o establecer otras obligaciones, incluyendo los individuos. Esta regla dotó a los medios de la posibilidad de contraer contratos con capital mixto, e incluso con propiedad privada indirecta, aportándoles mayor autonomía.

La creciente comercialización de la economía les retribuyó una mayor autonomía financiera y gerencial, y reorientó sus contenidos hacia la captura competitiva de intereses de consumo en nuevos targets. La publicidad no solamente constituyó una condición necesaria para la sobrevivencia económica de los medios, también fue un mecanismo que activó de forma importante el mercado y los nuevos hábitos de consumo de las audiencias, [12], tentando de forma provocadora los límites ideológicos del régimen político. 


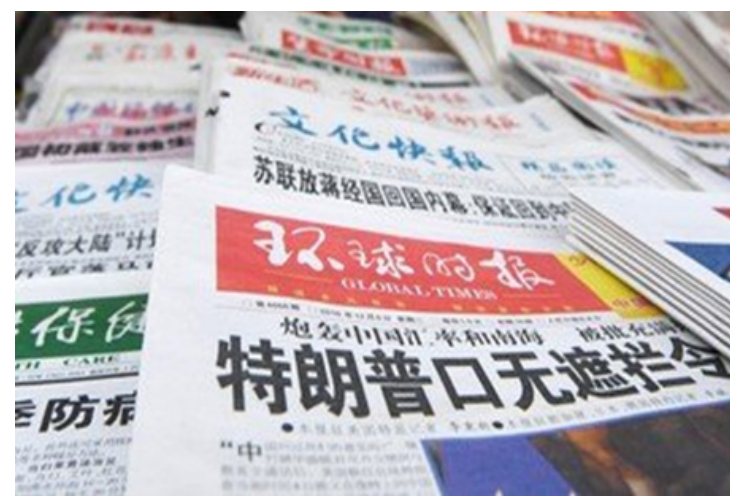

Un segundo impacto importante de la reforma económica fue sobre los contenidos de las publicaciones y los programas, pues una gran mayoría de organizaciones de medios respondieron a las nuevas fuerzas competitivas del mercado girando su programación al entretenimiento y noticias ligeras "soft" para estimular la curiosidad del público. Esta nueva variedad de contenidos permitió que nuevas voces y formatos llegaran al público, reduciendo el margen de influencia de la ideología comunista en la vida diaria de los individuos. El ímpetu económico de la reforma estimuló el desarrollo de la industria de la publicidad como indispensable recurso de ingresos, primero como suplemento y finalmente como sustituto de los subsidios que muchos medios recibían del partido/estado chino.

En tercer lugar, las reformas estimularon la liberalización de los medios fomentando su predictibilidad y estabilidad. Para obtener la estabilidad el estado estableció agencias burocráticas reguladoras (por ejemplo la Administración Estatal de Prensa y Publicaciones) que hicieron más predecible la relación estado-medios, lo que constituyó un incentivo importante a la inversión de capitales privados. Sin embargo, la regulación de medios se convirtió en un ejercicio más tecnocrático que ideológico, reflejando la creciente racionalización de un estado burocrático. Las nuevas reglas, aunque políticamente conservadoras, se configuraron, al menos en parte, por la lógica del mercado (Chan \& Qui; 2002).

Las reformas económicas inciden en la liberalización al provocar un cambio importante en la profesionalización periodística, orientando el reportaje informativo a las demandas por objetivos del mercado; su correlato fue un aumento de los periodistas jóvenes, más escépticos y mejor preparados con una creciente demanda: mayor independencia editorial del partido/estado chino. La creciente improvisación entendida como las prácticas periodísticas no rutinarias que los periodistas diseñaron, implementaron y justificaron erosionaron y debilitaron la hegemonía del sistema dominante asociado a la ideología comunista, al provocar una tensión entre los conflictivos roles periodísticos que deben cumplir: servir tanto a la élite política como al mercado; tensión para la cual no existían rutinas previstas ni adecuadas soluciones. Este proceso de improvisación dotó de mayor autonomía el trabajo periodístico.

La creciente internacionalización de los medios ha impactado en las últimas décadas el sistema de medios chinos consecuencia de relaciones reguladas o procesos de infiltración sin el consentimiento de las autoridades. La infiltración es resultado del poder liberalizante de ciertas tecnologías, específicamente cuando programas extranjeros son distribuidos en 
el país sin control de las autoridades gubernamentales chinas; por ejemplo, las señales de radio y televisión de Hong Kong y Taiwán en ciertas provincias chinas, o los efectos de la televisión satelital en las audiencias domésticas, ejerciendo una presión competitiva sobre los medios locales, los cuales responden imitando sus formatos y estilos.

El impetuoso mercado negro en China distribuye exitosamente grandes cantidades de contenidos extranjeros para el mercado doméstico. Es de esperar que el liderazgo reciente de China en la liberalización del comercio mundial abone a una flexibilización en los controles del mercado mediático interno, incluyendo sus sofisticados filtros para los contenidos on-line.

A pesar de estos avances, la liberalización de medios durante los años 80' y 90' fue relativa, reflejando los dramáticos giros de la política nacional en general, lo que permite definirlos como un sistema de medios autoritarios parcialmente liberalizados (Chan \& Qui; 2002) o en transición del totalitarismo a un autoritarismo de mercado (Winfield \& Peng; 2005). Consecuentemente, durante el periodo 1979-1989, el pensamiento liberal fue tolerado o suprimido en conexión con las ganancias y pérdidas de las fracciones de poder, aunque los factores económicos no son aún las fuerzas directivas de la transición del sistema de medios, la definición totalitaria previa de los medios como instrumentos de la lucha de clases fue oficialmente suprimida (Winfield \& Peng; 2005), y el control político varió entre los tipos de medios, géneros [13], y localizaciones geográficas. El control fue más relajado en medios no oficiales, información apolítica, y zonas alejadas de los centros de poder.

Al analizar la relación estado-medios-públicos en China, Zhao (2012) propone recuperar los fundamentos culturales e ideológicos históricos del estado al comunismo, el nacionalismo y el desarrollismo, los cuales han intervenido históricamente en la ideología del Partido Comunista Chino (PCCh). Es relevante la recuperación del principio Confucionista de la responsabilidad del estado para inculcar valores compartidos a través de la instrucción moral, y su ejercicio como medio de control social (2012: 152).

Durante un primer periodo revolucionario Maoísta (1940-1975), la prensa china fue parte de un sistema general de movilización ideológica y propaganda del partido de estado, pero a partir de 1978 el PCCh logró un consenso ideológico alrededor de la reforma y la apertura. En el sector de medios e industrias culturales, el estado drásticamente redujo su rol subsidiando las operaciones de éstos, y le ofreció paulatinamente seguridad jurídica a los derechos de propiedad privada y la maximización de ganancias. No obstante, la orientación ideológica del partido de estado continuó articulando diferentes versiones de marxismo y socialismo, cuyo fin era la construcción de "un socialismo con características chinas", y los medios debían proveer la guía moral a la población y la ingeniería del desarrollo económico y del cambio social.

Las políticas estatales hacia el sector han sido complejas y diferenciadas (Zhao, 2012), pues tanto la producción y distribución de noticias y contenidos informacionales, como la propiedad de los grandes medios impresos y de transmisión, continúo siendo monopolio del Estado. Sin embargo, la propiedad completa o parcial de las áreas periféricas de las industrias de medios y culturales, incluyendo la producción de filmes, entretenimiento para 
televisión y publicidad, así como la distribución audiovisual, fueron abiertas al financiamiento del capital nacional e internacional.

Esta dualidad garantiza al Estado retener el control sobre el sistema de medios y su orientación ideológica, sin monopolizar la producción y distribución, especialmente en entretenimiento, estilo de vida y áreas de negocios. [14]. Los propios órganos del partido de estado dirigen el proceso de comercialización, canalizando los mecanismos de mercado al interior de las estructuras existentes, y desarrollando diversos mecanismos de control como la implementación de un registro de licencias y de certificación profesional periódica, un código de ética profesional para los periodistas y un programa de entrenamiento "ideológico" obligatorio (Zhao, 2012). [15].

No obstante, la creciente comercialización de la economía dotó a los medios de una mayor autonomía financiera y gerencial, y una importante reorientación de sus contenidos hacia la captura competitiva de intereses de consumo en nuevos targets [16], y la gestión estructural de los medios desde agencias especializadas de gobierno permitió una relación de mayor predictibilidad y estabilidad entre el Estado y los medios y un incentivo importante a la inversión de capitales privados. Así, la regulación de medios adquirió un creciente énfasis tecnocrático regido por la lógica del mercado, retando el componente ideológico partidista (Winfield \& Peng, 2005; Chan \& Qui, 2002). Las nuevas prácticas periodísticas comienzan a cambiar la autopercepción de los periodistas del rol de legitimación ideológica del partido a algún tipo de servicio público como un grupo profesional diferenciado con una función social distinta (Zhao, 2012; Tong \& Sparks, 2009).

Un estudio reciente muestra la contingente complejidad que define las sistemáticas negociaciones y los roles colaborativos del periodismo de investigación chino, así como la persistente adaptabilidad y resiliencia del régimen Comunista (Repnikova, 2017). Para la autora, al aceptar las reglas de la política de medios definida por los funcionarios del partido, el periodismo de investigación conoce las "fronteras de lo permisible", evitando centrar la atención en los temas que cuestionan la responsabilidad pública del Estado y su legitimidad y asumen su rol consultivo y de vigilancia de las autoridades locales, por lo que contribuyen a una "improvisada gobernanza" (Repnikova, 2017).

En el caso latinoamericano, el escenario de reformas mediáticas se ha caracterizado por la persistencia de antiguas culturas periodísticas, los intereses estatales y corporativos, la pobre gobernanza y la polarización ideológica (Lugo-Ocando \& García Santamaría; 2015).

\section{Dos categorías intrínsecamente "comunicativas": transición política y nuevos movimientos sociales}

J. Downing (1996) en su análisis de las democratizaciones en la URSS, Polonia y Hungría, reconoce que la dimensión cultural y mediática ha sido abordada de forma superflua, tanto en los estudios de transiciones como en las teorías sobre los actores que promueven estos cambios, los (nuevos) movimientos sociales, a pesar del énfasis intrínseco de sus dinámicas comunicativas. 
La transición es un proceso dinámico de redefinición de las reglas del juego político a partir de interacciones estratégicas de actores implicados en un cambio de régimen. Esta dinámica presupone la presencia de opciones alternativas preferibles como una inclinación real para los individuos aislados -una contra hegemonía- y la creación de instituciones específicas que afectan la capacidad de los diversos grupos para satisfacer sus intereses a partir de transacciones contingentes (Przeworski; 1994).

El primer umbral crítico en la transición hacia la democracia es el movimiento iniciado por algún grupo del bloque gobernante para lograr apoyo de fuerzas exteriores a él, y las decisiones de liberalizarlo combinan elementos inspirados desde "arriba" y desde "abajo" (Przeworski; 1994). La liberalización es resultado de una interacción entre la aparición de fisuras en el régimen autoritario y la organización autónoma de la sociedad civil. Sin embargo, el propósito liberalizador (o proyecto alternativo) debe ser lo suficientemente creíble como para provocar un cambio en la estrategia de los demás actores (O'Donnell y Schmitter, 1994).

En otras palabras, el inicio de la liberalización de un régimen autoritario/totalitario presupone una fractura en términos de (auto) percepciones, expectativas de cambio y recursos al interior de la élite gobernante y la necesaria existencia de un nivel de autonomía, recursos asociativos y de movilización en sectores de la sociedad civil opuestos al régimen, por tanto, interesados en la elaboración (y justificación) de proyectos colectivos para un futuro alternativo creíble.

A pesar de la sofisticación del análisis estratégico, la transición política implica la construcción de un escenario de negociación donde actores interesados establecen acuerdos sobre las condiciones mínimas mutuamente ventajosas para la construcción de un nuevo escenario político. Como acertadamente reconoce Downing, esta dinámica, aún al interior de la élite política autoritaria "arriba" implica inobjetablemente un componente dialógico -un intercambio de información- o comunicacional en el proceso de elección estratégica de los actores que ha sido sugerida, pero no examinada (1996).

Respecto a la sociedad civil la literatura de transición reconoció la capacidad de ciertos liderazgos y prácticas culturales en espacios informales autónomos "abajo" como cafés, tertulias literarias, galerías de arte independiente, librerías, etc. para crear espacios de discusión, debate y elaboración de propuestas alternativas, desafiando así las reglas autoritarias del régimen y constituyéndose en un emergente sector disidente.

Por otro lado, en la larga tradición analítica sobre los movimientos sociales, aún desde la perspectiva de Oportunidades Políticas que define a estos movimientos como sistemas de acción que operan en un campo de posibilidades y límites, donde su fundamento es el nexo concreto entre orientaciones y oportunidades/constricciones sistémicas del proceso político, se pueden visualizar interesantes elementos sobre los fundamentos culturales y simbólicos de la acción colectiva. 
Para Tarrow, una de las principales tareas de la organización del movimiento es encontrar símbolos que resulten lo suficientemente conocidos para movilizar a la gente que lo rodea, pues "mantener la integridad del movimiento frente a las exigencias de la cultura heredada es la otra cara del problema" (1997: 209). Esta premisa lo conduce a pensar la necesidad de conceptualizar el papel del entorno simbólico-cultural, completamente subvalorado por la tradición teórica anterior, definiendo la categoría de marco interactivo como los dispositivos de acentuación que subrayan y adornan la gravedad y la injusticia de una situación social o redefinen como injusto o inmoral lo que previamente era considerado desafortunado, aunque tal vez tolerable (1997: 215) [17].

Una tarea fundamental de los movimientos sociales será entonces la de señalar agravios, vincularlos a otras ofensas y construir marcos de significados más amplios que puedan encontrar eco en la predisposición cultural de una población y transmitir un mensaje uniforme a quienes ostentan el poder y a otros estamentos. En palabras de Tarrow, la actividad clave de los movimientos sociales consiste en "inscribir agravios en marcos globales que identifican una injusticia, atribuir la responsabilidad de la misma a otros y proponer soluciones" (1997: 215).

Pero el autor de mayor énfasis en el aspecto micro-cognitivo y cultural de la acción colectiva fue Alberto Melucci, quien reconoce que a diferencia de los enfoques de la movilización de recursos, que evitan el grado micro y reducen toda acción colectiva a su dimensión política y organizacional, los conflictos sociales contemporáneos no son sólo políticos, pues afectan la producción cultural del sistema (1999: 47).

Los individuos operando conjuntamente construyen su acción mediante inversiones "organizadas"; es decir, definen en términos cognoscitivos, afectivos y relacionales el campo de posibilidades y limites que perciben, y al mismo tiempo, activan sus relaciones para darle sentido al "estar juntos" y a los fines que persiguen. Este proceso de construcción y negociación del significado de la acción colectiva, llamado identidad colectiva, enfatiza en la necesidad de un grado de identificación que es precondición para cualquier cálculo costobeneficio y para la percepción de la injusticia como tal. [18].

En esta misma dirección K. Glenn III (2001) explica el éxito de los movimientos civiles en Europa del Este en 1989, señala que éstos presentaron demandas sobre injusticia, identidad y agencia en sus intentos por movilizar redes de apoyo. Estos marcos de acción colectiva (framing) son definidos como interpretaciones de eventos estratégicamente orientados a través de los cuales los activistas de los movimientos sociales buscan apoyo y movilización, vinculando la participación con el esquema cognitivo o compartido de patrones internalizados de ideas y sentimientos que media entre el manejo individual y el ambiente político externo.

Para el autor, un sentido de injusticia debe ser combinado con una identidad, lo cual definirá el "nosotros versus ellos", y un sentido de agencia o conciencia que los cambios son posibles y que la acción individual puede ser efectiva. Como se aprecia, el framing juega un rol esencial no solo para persuadir a los individuos a unirse al movimiento bajo favorables 
oportunidades, sino que alinea las demandas de los retadores con las identidades de redes preexistentes, redefiniendo los roles existentes al interior de las organizaciones establecidas sobre la base de una emergente identidad activa (K. Glenn; 2001).

\section{Conclusiones}

Las transformaciones en las dinámicas políticas de estos regímenes generalmente implican procesos de liberalización en la producción y distribución de los flujos informativos. Sin embargo, las nuevas prácticas periodísticas no necesariamente asumen los principios liberales del equilibrio y la autonomía editorial frente a las nuevas elites. Como demuestra la evolución de la Rusia post-soviética posterior a 1992, las reformas de liberalización económica y la competencia política y electoral produjeron un cambio institucional con resultados inciertos: la emergencia de nuevas elites económicas y políticas coludidas en pos del capital económico y político, con un creciente control sobre la regulación y los contenidos de los medios.

En este contexto de control político, las prácticas periodísticas rechazan la idea de "objetividad" y "balance" y el periodista se auto-concibe como un jugador político al servicio de patrocinadores financieros y políticos y no en su función de watchdogs o vigilante del Estado. Finalmente, el aumento del consumo implicó para las audiencias asumir valores como el éxito individual, el hedonismo y la apatía política.

En regímenes autoritarios/totalitarios como China, las transformaciones económicas les han imprimido cierto dinamismo a las prácticas periodísticas, modificando la autopercepción del periodista chino de la función social de legitimador ideológico del partido (Zhao, 2012; Tong \& Sparks, 2009). Sin embargo, el periodismo de investigación, resultado de la liberalización económica y la nueva política de medios del PCCh, ha asumido un rol consultivo y de vigilancia de las autoridades locales, evitando centrar la atención en temas que confrontan al estado y su legitimidad. Este rol colaborativo del nuevo periodismo chino ha contribuido a la persistente adaptabilidad y resiliencia del régimen Comunista (Repnikova, 2017).

Por su parte en las democracias híbridas latinoamericanas, las reformas mediáticas reproducen rasgos de las antiguas culturas periodísticas, la fusión de los intereses estatales y corporativos, los problemas de gobernanza y la polarización ideológica (Lugo-Ocando \& García Santamaría; 2015).

El artículo llama la atención sobre la necesaria recuperación del componente dialógico y comunicacional implícito en las dinámicas de negociación y construcción de consensos que los procesos transicionales implican, así como el importante papel del entorno simbólicocultural en la definición de los marcos interactivos que definen un agravio público. A partir de estos componentes se articula la dimensión relacional y organizativa que define la identidad colectiva (Melucci, 1996). El éxito liberalizador de un movimiento social depende entonces de cómo éstos presentan demandas sobre injusticia, identidad y agencia en sus intentos por movilizar redes de apoyo. He ahí la importante función del periodismo y los medios en estos regímenes. 


\section{Bibliografía}

Barrera, C. \& Zugasti, R. (2006), "The role of the press in time of transition: the building of the Spanish democracy 1975-1978", en Voltmer, K. Mass Media and Political Communication in New Democracy, New York: Routledge.

Chan, J. M. \& Qui, J. L. (2002), “China: media liberalization under authoritarianism”, en Price \& Rozumilowicz, Media Reform. Democratizing the media, democratizing the state, London: Routledge.

Curran, J. \& Jin Park, M. (2000), De-Westernizing Media Studies, New York: Routledge

Downing, J. D. H. (1996), Internationalizing Media Theory. Transition, Power, Culture, London: SAGE Publications.

Esser, F. \& Pfetsch, B. (2004), Comparing Political Communication. Theories, Cases, and Challenges, United Kingdom: Cambridge University Press.

Glenn III, J. K. (2001), Framing Democracy. Civil Society and Civic Movements in Eastern Europe, Stanford, California: Stanford University Press.

Gross, P. \& Jakubowicz, K. (2013), Media Transformations in the Post-Communist World. Eastern Europe's Tortured Path to Change, United Kingdom: Lexington Books.

Gunther, R. \& Mughan, A. (2000), Democracy and the Media. A Comparative Perspective, United Kingdom: Cambridge University Press (CUP).

Hallin, D. \& Mancini, P. (2012), Comparing Media Systems beyond the Western World, United Kingdom: Cambridge University Press (CUP).

Hallin, D. \& Mancini, P. (2007), Sistemas Mediáticos Comparados, Barcelona: Editorial Hacer. Hedwig de Smaele, (2006), "In the name of democracy: the paradox of democracy and press freedom in post-communist Russia", en Voltmer, K, Mass Media and Political Communication in New Democracies, New York: Routledge.

Lugo-Ocando, J. \& García Santamaría, S. (2015), "Media, Hegemony and Polarization in Latin America”, en Zielonka, J. (2015).

Vartanova, E. (2012), “The Russian Media Model in the Context of Post-Soviet Dynamics", en Hallin, D. \& Mancini, P. (2012).

Voltmer, K. (2006), Mass Media and Political Communication in New Democracies, New York: Routledge.

O’Donnell, G. \& Schmitter, P. (1994), Transiciones desde un gobierno autoritario. Perspectivas comparadas, Barcelona: Paidós, Vol. 3 y 4. 
Price, M. E. \& Rozumilowicz, B. \& Verhulst (2002), Media Reform. Democratizing the media, democratizing the state, London: Routledge.

Przeworski, A. (1994), "Algunos problemas en el estudio de la transición hacia la democracia”, en O'Donnell, G. y Schmitter, P., Transiciones desde un gobierno autoritario. Perspectivas Comparadas, Barcelona: Paidós, Vol. 3, pp. 79-104.

Meng, B. \& Rantanen, T. (2015), "A Change of Lens: A call to compare the media in China and Russia", Critical Studies in Media Communication, Vol 32, No. 1, pp. 1-15.

Melucci, A. (1999), Acción colectiva, vida cotidiana y democracia, México: El Colegio de México.

Oates, S. (2007), "The Neo-Soviet Model of the Media", Europe-Asia Studies, Vol. 59, No. 8, Dec, pp. 1279-1297.

Randall, V. (1993), "The Media and Democratization in the Third World," Third World Quarterly, Vol. 14, No. 3, pp. 625-646.

Reig Cruañes, P. (2014), “La prensa en la transición democrática: ni 'motor del cambio' ni 'parlamento de papel'”, en Guillamet, J. \& Salgado, F., El periodismo en las transiciones políticas. De la Revolución Portuguesa y la Transición Española a la Primavera Árabe, Madrid: Biblioteca Nueva.

Repnikova, M. (2017), Media, Politics in China: Improvising Power under Authoritarism, United Kingdom: Cambridge University Press.

Roudakova, N. (2012), "Comparing Processes: Media, "Transition”, and Historical Change", en Hallin, D. \& Mancini, P. (2012).

Roudakova, N. (2008), "Media, political clientelism: lessons from anthropology", Media, Culture \& Society, Vol. 30 (1), pp. 41-59.

Tarrow, S. (1997), El poder en movimiento. Los movimientos sociales, la acción colectiva y la política, Madrid: Alianza Editorial.

Tong, J. \& Sparks, C. (2009), "Investigative Journalism in China Today", Journalism Studies, Vol. 10, No. 3, pp. 337-352.

de Smaele, (2013), adrid. .( eds.), El periodismo en las transiciones poltario. Perspectivas comparadas, Barcelona, PaidWinfield, B. H. \& Peng, Z. (2005), "Market or Party controls. Chinese Media in Transition", The International Journal for Communication Studies, SAGE Pub. pp. 255-270.

Zao, Yuezhi (2012), “Understanding China's Media System in a World Historical Context”, en Hallin, D. \& Mancini, P. (2012). 
Zielonka, Jan (2015), Media and Politics in New Democracies: Europe in a Comparative Perspective, United Kingdom: Oxford University Press.

\section{Notas}

[1] Entre los factores de nivel macro que condicionan el mensaje de los medios y sus patrones de circulación está la cultura política, la estructura de la sociedad, los medios y las instituciones de gobierno, las normas que regulan la relación entre periodistas y políticos, las prácticas regulatorias y el nivel de desarrollo tecnológico de la industria de la comunicación; el nivel micro apunta a factores subjetivos de la recepción como las proclividades actitudinales o culturales, los niveles de educación y la complejidad cognitiva, el grado de exposición a cada medio, además de la presencia de subculturas autónomas o redes de asociación secundaria que tienen profundas implicaciones para la receptividad de la comunicación política (Gunther \& Mughan; 2000).

[2]Según los autores, la relativa estabilidad de las sociedades occidentales ofrece la ilusión de que ellas representan el punto final de la historia hacia el cual se mueven los países no occidentales (Meng \& Rantanen; 2015: 4).

[3]Partiendo de la premisa que los regímenes híbridos son formaciones políticas en constante cambio histórico en dirección incierta, Roudakova asume las organizaciones de medios como sitios donde se superponen procesos interconectados como el ejercicio del poder político, las dinámicas ideológicas, la acumulación de capital económico, las tensiones y luchas sociales, la producción de representaciones culturales, y la creación de identidades profesionales (2012: 262).

[4] "No importa el grado de obligación o devoción que hubiera en ello, o si todos esperaban en la intimidad de sus redacciones el momento de la libre expresión para ser felices. Lo que importa es el hecho de que el resultado, lo que la gente leía, veía y oía, era siempre un sonsonete de legitimidad, acierto y bienaventuranza para el régimen dictatorial y sus protagonistas. Con pequeñas disonancias locales, es cierto, descontextualizadas y tímidas, que debían buscarse entre líneas y solo los más avisados eran capaces de descubrir. La búsqueda de ocultas significaciones críticas en los textos llegó a constituir una habilidad muy extendida en los círculos de desafectos" (Reig Cruañes; 2014: 168).

[5] "Los términos con mayores referencias explícitas en 441 editoriales españolas publicadas entre 1975-1978 fueron: democracia, libertades civiles, autonomía, amnistía, elecciones y referendo, concordia y reconciliación, autoridad y orden, Franco, franquismo y/o Guerra Civil" (Barrera y Zugasti; 2006: 28).

[6] Hedwig de Smaele enumera una larga lista de acciones cometidas por B. Yelstin en nombre de la democracia: prohibir un número de diarios que inequívocamente condenaron el golpe de estado de Agosto de 1991 y Octubre de 1993, y abiertamente incorporó a los medios masivos a su campaña de re-elección como presidente en 1996; además, le 
presentó su heredero a las audiencias y electores en el otoño de 1999. "Es obvio que B. Yelstin permitió más libertades que sus predecesores, y nunca dudó sobre lo que consideró los derechos a permitir (o prohibir) libremente a su discreción. En cambio, esperó lealtad de los medios para apoyar sus reformas." (2013: 137).

[7] Sarah Oates reconoce que existe una "guerra" de leyes en Rusia afectando al sector de medios masivos pues el presidente puede decretar temas que entren en conflicto con leyes aprobadas en el parlamento; y más confuso aún, las regiones pueden regular los medios localmente e imponer leyes contradictorias sobre los medios y periodistas locales. Esta contradicción es ventajosamente aprovechada por políticos nacionales y locales que amenazan a los medios con el cierre por coberturas adversas (2007).

[8] "Las noticias son significativas para dirigir más que para describir; para enfatizar los bienes de la sociedad soviética e ignorar lo malo. Los medios envían un claro y nada ambiguo mensaje sobre los valores soviéticos, dando a la audiencia un sentido de alegría y orgullo en su sociedad. (.......). En particular, ellos encuentran importante que los medios jueguen un inspirador rol sobre las fortalezas de la Federación Rusa desde su periodo de fundación. Ello explica por qué los rusos consistentemente eligen el Canal 1 estatal como su favorito y más confiable recurso de noticias, generalmente más confiable que otras instituciones políticas en el país" (Oates; 2007: 1295-1296).

[9]Para la investigadora, "los periodistas rusos deben adherirse a la agenda de noticias del medio forzados por los controles internos del propio medio: al tolerar menos oposición el Kremlin, el medio desafiante perderá todo el apoyo financiero y se le aplicará discrecionalmente las leyes; derivado de ello, periodistas que den cobertura a temas controversiales chocarán con editores y publicistas cautelosos que los pueden censurar" (Oates; 2007: 1287).

[10]En palabras de Vartanova, después del colapso del sistema de prensa soviético, la nueva prensa local y regional Rusa se define por su alta orientación comercial, sensacionalista e instrumentalizada por las alianzas informales entre el estado y las elites empresariales (2012: 127).

[11]Entre las regulaciones y controles del estado ruso sobre los medios están el registro obligatorio de medios masivos, las restricciones sobre difamación, las regulaciones antiterroristas, el nombramiento de personal directivo de los canales de televisión importantes por el Ministerio de Medios, la dependencia financiera de los subsidios del estado o patrocinadores corporativos semi-estatales, o acceder a facilidades como editoriales, imprentas, acceso a transmisiones o satélites cuya licencia deben obtener de agencias estatales (Hedwig de Smaele, 2013).

[12]Para Winfield \& Peng los ingresos por publicidad, circulación y apoyos de inversión dotaron a los medios de una mayor independencia financiera del gobierno, y al mismo tiempo, de una mayor dependencia de las audiencias (2005: 260).

[13] En géneros como el cine, que en el periodo pre-reforma fueron estrictamente 
controlados por el CCP, han presentado un relajamiento de estos controles lo que les ha permitido acercar sus contenidos a los géneros internacionales e importar programas de Hong Kong, Taiwán, Europa y Hollywood (Chan \& Qui; 2002).

[14]Zhao reconoce que la comercialización de medios controlada por el partido de estado lo habilita para dividir las tareas entre dos segmentos primarios de prensa: los órganos tradicionales partidistas, los cuales están orientados a la comunicación intra-partidista entre el líder y la unidad de sus bases, y los periódicos de apelación de masas, los cuales le sirven al Partido Comunista Chino como medio para renegociar y rearticular los términos de su hegemonía con las crecientes clases medias urbanas (2012: 158).

[15]La codificación de la ética profesional dirigida por el Estado, según Zhao (2012), permite al régimen transformar su autoridad política en autoridad moral como instrumento para sujetar a los periodistas a un sistema nacional de certificación profesional, "renovables cada cinco años, y sujetos a una revisión anual". Además, los periodistas deben tomar un programa de entrenamiento en ideología oficial, regulaciones y políticas de medios, ética periodística, teoría marxista de prensa, y tópicos relacionados (p. 163).

[16]Según Zhao, la comercialización ha contribuido al desarrollo de dos poderosos nichos de mercados de elite: la prensa orientada a la elite intelectual, y los papers especializados dirigidos a un grupo de influyentes empresarios de la naciente clase empresarial China; ambos con claras orientaciones liberales y tecnocráticas, a diferencia de los funcionarios partidistas, lectores de los órganos tradicionales del partido (2012).

[17]El concepto original de marco interactivo citado por Tarrow fue desarrollado por Snow y R. Benford: "esquema interpretativo que simplifica y condensa el 'mundo de ahí afuera' puntuando y codificando selectivamente objetos, situaciones, acontecimientos, experiencias y secuencias de acciones dentro del entorno presente o pasado de cada uno" (Tarrow; 1997: 214).

[18]Para Melucci, la propensión de un individuo a implicarse en la acción colectiva está ligada a la capacidad diferencial para definir una identidad, esto es, al acceso diferencial a los recursos que le permiten participar en el proceso de construcción de una identidad. Entonces, el grado de exposición de un individuo a ciertos recursos (cognoscitivos y relacionales) influye en su posibilidad o no, de entrada, en el proceso interactivo de construcción de una identidad colectiva (1999: 66-67).

\section{Para citar este artículo}

Rodríguez, C. (2020). Aproximaciones teóricas a la relación entre Estado, Medios y Públicos: España, Rusia y China. Revista Luciérnaga Comunicación. Vol. 12 Núm. 24. Pp. 90-110. https://doi.org/:10.33571/revistaluciernaga.v12n24a6

OJS: https://revistas.elpoli.edu.co/index.php/luc/issue/archive

Link: https://www.politecnicojic.edu.co/index.php/revista-luciernaga 